\title{
An Enhanced Performance Measurement in Spread
}

\author{
M.Selvi R.Balakrishna
}

\begin{abstract}
A SPREAD programme is anticipated to enhance the safe data supply and delivery in a MANET. The main aim of SPREAD is to divide a message into many parts by secret sharing and sends them via various autonomous paths to the end point. This paper focuses and highlights the spread design and its performance metrics. SPREAD is considered to be more protected and also possess comparatively high amount of reliability because of presence of redundancy without compromising safety. Simulation outcomes validate the feasibility of the SPREAD method and display the efficiency and performance metrics.
\end{abstract}

Keywords - SPREAD, MANET, redundancy, reliability and feasibility

\section{INTRODUCTION}

Mobile ad hoc networks (MANET) were developed to control the protocols and essential parameters of ad hoc wireless systems in the 1980s. Since, the research on defence instrumentation was widely funded across the globe; a task force in the Internet Engineering recognized MANET as the probable program for the future. The main idea of the task force is to ensure MANET to deliver improved harmonized routing functionality to sustain a selforganizing mobile networking structure. Defence adoption, collective and distributed computing, crisis handling, wireless mesh, wireless sensor networks and hybrid wireless network design are some of the important utility of ad hoc wireless networks. The key problems which shall affect the plan, operation, and work of an adhoc wireless network are multicasting, pricing system, the excellence of service, energy management, and service.

\section{Adhoc network diagram}

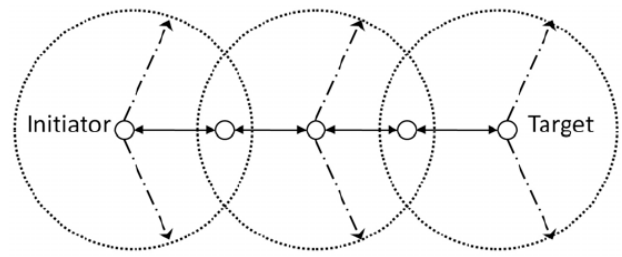

SPREAD statistically improves the data confidentiality in a MANET. The SPREAD scheme performs with the assumption of having secret information. The adversary could easily follow the communication by any one of the nodes along the path if the message is send the through the single path. However, the enemy would have to confront all the information bits and the sequence to understand the communication if they are split into multiple pieces, and send through numerous self-determining paths. The security of SPREAD system can be attained by the above process.

\section{SPREAD ARCHITECTURE}

The two fundamental principles of SPREAD are secret sharing and multipath routing. The secret communication is separated into several parts by secret sharing systems. This system allows the splitting of secret messages into many parts and wants the critical information of some scattered parts to rebuild the original secret information. Further, the scheme guarantees fewer chances of secret recovery to the owner if lesser threshold number of shares are recognised. No secret message can be ascertained if an outsider knew nothing about the system. The secret sharing system is competent and totally protected as the defence it delivers is independent of the computing period or influence that a rival may bring to challenge the system.

Multipath routing is described as traffic dispersion which has been one of the significant developments in the field of routing. A better connected network would have many directions between the initiator and endpoint. This routing is constructed on the single shortest path which provides the base node an option on some specified time of several paths to a particular target by captivating gain of the connectivity redundancy. Many types of research on multipath routing have been conducted for a number of network monitor and management aspects in different networks. This routing method is used to pool bandwidth, reduce late arrival, to sustain service quality, to relax the traffic congestion, to advance the network crowding and fault tolerance. Multipath routing is highly useful in managing normal topological variations and improves flexibility to MANET in our SPREAD scheme. The safety achieved in the secret sharing system could be enhanced while the message is diffused across the network.

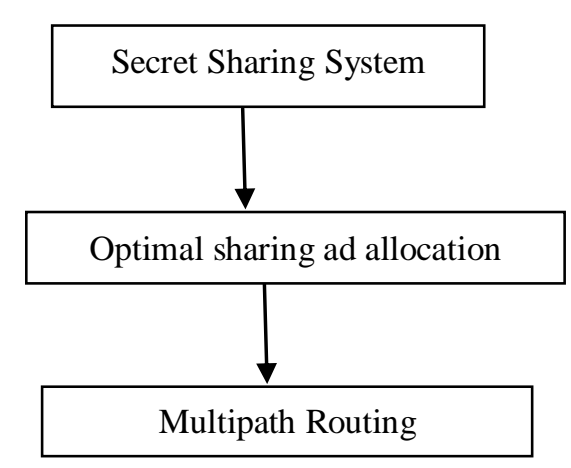

Revised Manuscript Received on July 18, 2019.

M.Selvi, Department of Computer Science Research Scholar,VTU Bangalore,India(E-mail:.Selvim1983@gmail.com)

Dr.R.Balakrishna, Department of Computer Science RajaRajeswari Engineering College Bangalore,India (E-mail:.rayankibala@gmail.com) 


\section{AN ENHANCED PERFORMANCE MEASUREMENT IN SPREAD}

\section{SIMULATION RESULTS}

The simulation outcomes figure out the efficiency of the spread scheme in implementing the information confidentiality which is provided in this part. An adhoc network is simulated with 100 nodes and arbitrarily engaged in a 1000 meter square area. Each simulation will have equal spread range of individual node and varies in different simulations. Further, the simulation outcomes are averaged over twenty randomly installed networks. Evaluation of application level performance metrics like packet delivery ratio, packet delay, throughput, time complexity and space complexity are ascertained.Packet delivery ratio is the piece of packets that are accepted at corresponding end point over those

sent at the source. Packet delay is between a packet deliver at the source received at the end point. Throughput is the number of routing packets accepted successfully by individual routing protocol. Packet loss is the failure of single or more transmitted packets to reach their destination. Time complexity refers to the quantification of time to run as a function of the amount of input. Space complexity is a function explaining the volume of memory space an algorithm considers in terms of the amount of input to the algorithm. Energy is understood as the capacity of physical system to accomplish work and residual energy.

\section{A.Packet Delivery ratio result}

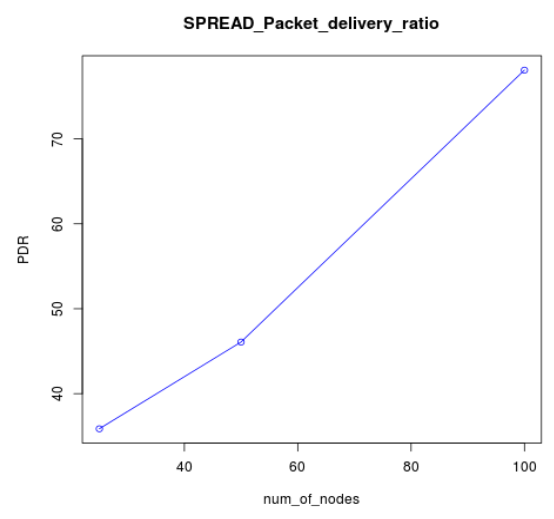

B. Throughput result

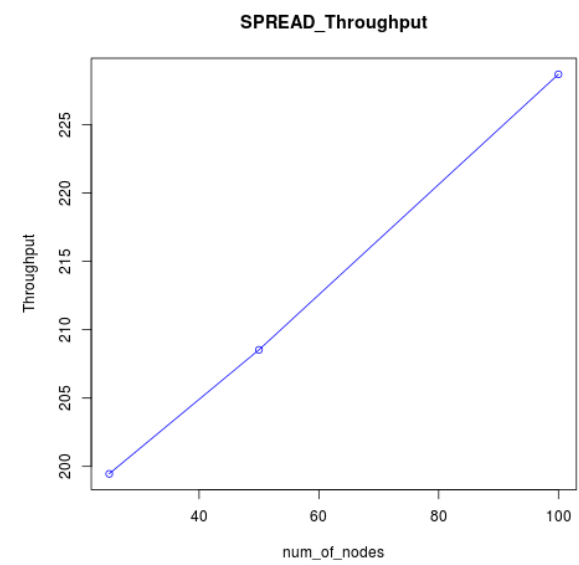

\section{C.Delay result}

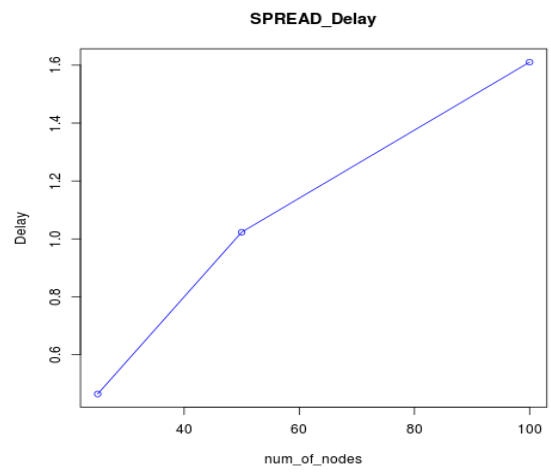

D.Packet Loss result

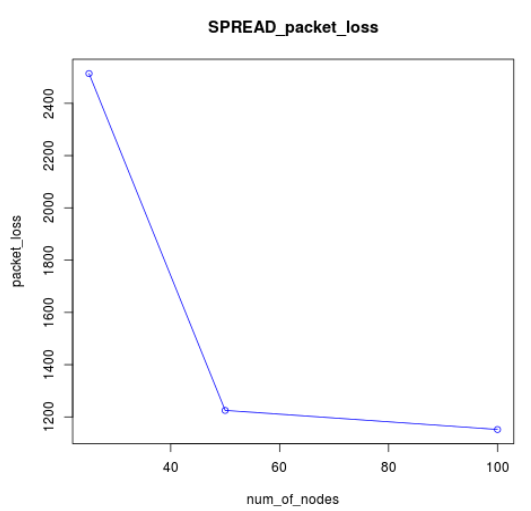

E.Time Complexity result

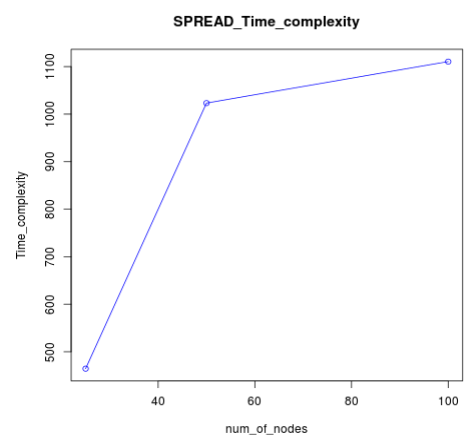

F.Space Complexity result

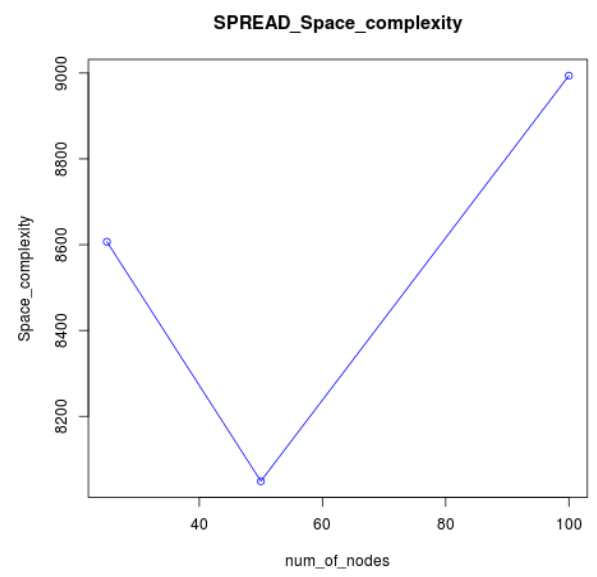

Published By: 


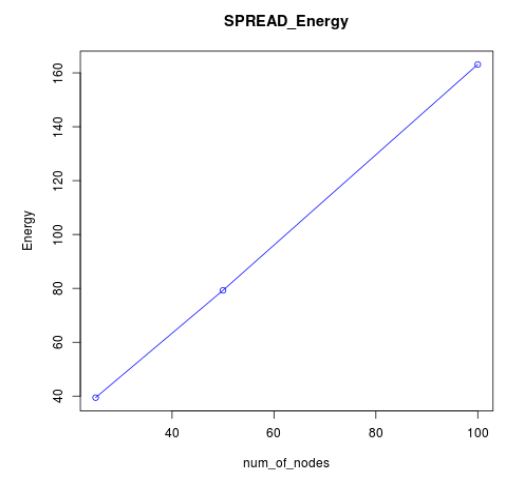

H. Residual Energy result

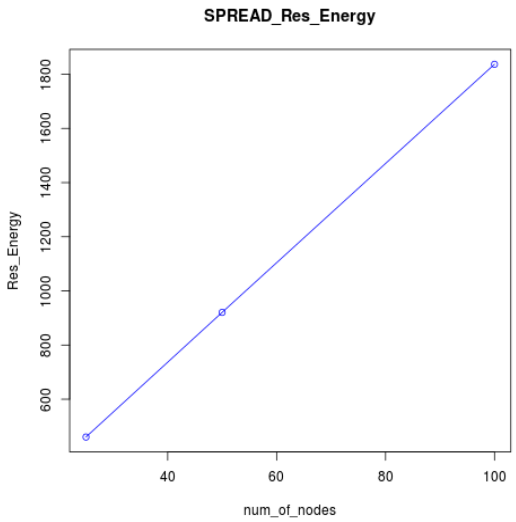

\section{CONCLUSION}

SPREAD is an effective means to improve the data privacy and designed to improve the reliability of the transmitted data, in contrast to the unbalanced wireless connection problem and frequent topological differences. The simulation results also display that the crucial improvised security system can be attained by SPREAD.

\section{REFERENCES}

1. Navdeep Kaur, Amandeep Kaur," Study of Mobile Ad-hoc Network", International Journal of Emerging Engineering Research and Technology Volume 3, Issue 5, May 2015, PP 8-12 ISSN 23494395 (Print) \&amp; ISSN 2349-4409 (Online)

2. M. Frodigh, P. Johansson, and P. Larsson,"Wireless ad hoc networking: the art of networking without a, Network," Ericsson Review, No.4, 2000.

3. Marco Conti, Body, Personal and Local Ad Hoc Wireless Networks, in Book The Handbook of Ad Hoc Wireless Networks (Chapter 1), CRC Press LLC, 2003.

4. Basagni, S., Conti, M., Giordano S., and Stojmenovic, I. (Eds.) “Ad Hoc Networking” IEEE Press Wiley, New York, 2003.

5. Sadiya Mirza, Sana Zeba Bakshi,"INTRODUCTION TO MANET", InternationalResearch Journal of Engineering and Technology(IRJET) e-ISSN: 2395-0056 Volume: 05 Issue: 1 |Jan-2018 www.irjet.net p-ISSN: 23950072 